\title{
THE
}

\section{Ethnicity as a moderator of motivational interviewing for incarcerated adolescents after release}

\author{
Mary Clair \\ University of Rhode Island
}

\author{
L.A.R. Stein \\ University of Rhode Island, larstein@uri.edu \\ Shayna Soenksen \\ University of Rhode Island \\ Rosemarie A. Martin \\ University of Rhode Island \\ Rebecca Lebeau \\ University of Rhode Island \\ Follow this and additional works at: https://digitalcommons.uri.edu/psy_facpubs \\ This is a pre-publication author manuscript of the final, published article.

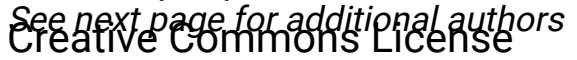

\section{(c) $($ †) $\Theta$}

This work is licensed under a Creative Commons Attribution-Noncommercial-No Derivative Works 4.0 License.

\section{Citation/Publisher Attribution}

Clair, M., Stein, L.A.R., Soenksen, S., Martin, R. A., Lebeau, R., \& Golembeske, C. (2013). Ethnicity as a moderator of motivational interviewing for incarcerated adolescents after release. Journal of Substance Abuse Treatment, 45(4), 370-375. doi: 10.1016/j.jsat.2013.05.006

Available at: https://doi.org/10.1016/j.jsat.2013.05.006

This Article is brought to you for free and open access by the Psychology at DigitalCommons@URI. It has been accepted for inclusion in Psychology Faculty Publications by an authorized administrator of DigitalCommons@URI. For more information, please contact digitalcommons-group@uri.edu. 


\section{Authors}

Mary Clair, L.A.R. Stein, Shayna Soenksen, Rosemarie A. Martin, Rebecca Lebeau, and Charles Golembeske 


\title{
Ethnicity as a moderator of motivational interviewing for incarcerated adolescents after release
}

\author{
Mary Clair, Ph.D. ${ }^{a, b},{ }^{\star}$, L.A.R. Stein, Ph.D.a,b,c, Shayna Soenksen, M.S. ${ }^{a}$, Rosemarie A. \\ Martin, Ph.D. ${ }^{c}$, Rebecca Lebeau, Ph.D. ${ }^{a}$, and Charles Golembeske, Ph.D. ${ }^{b}$ \\ Mary Clair: mclair@uri.edu \\ aSocial Sciences Research Center, University of Rhode Island, Kingston, RI 02881, USA \\ bThe Rhode Island Training School, Cranston, RI 02920, USA \\ ${ }^{\circ}$ Center for Alcohol and Addiction Studies, Brown University, Providence, RI 02906, USA
}

\begin{abstract}
Motivational interviewing (MI) has been found to be an effective treatment for substance using populations, including incarcerated adolescents. Although some studies suggest MI is more successful with individuals from minority backgrounds, the research remains mixed. The current study investigated the impact of ethnicity on treatment in reducing alcohol and marijuana use among incarcerated adolescents. Adolescents (14-19 years of age) were recruited from a state juvenile correctional facility and randomly assigned to receive MI or relaxation therapy (RT) $(N=$ 147; 48 White, 51 Hispanic, and 48 African American; 126 male; 21 female). Interviews were conducted at admission to the facility and 3 months after release. Results suggest that the effects of MI on treatment outcomes are moderated by ethnicity. Hispanic adolescents who received MI significantly decreased total number of drinks on heavy drinking days (NDHD) and percentage of heavy drinking days (PHDD) as compared to Hispanic adolescents who received RT. These findings suggest that $\mathrm{MI}$ is an efficacious treatment for an ethnic minority juvenile justiceinvolved population in need of evidence-based treatments.
\end{abstract}

\section{Keywords}

Adolescents; Incarceration; Motivational interviewing; Ethnicity; Alcohol

\section{Introduction}

\subsection{Adolescent crimes and substance use}

Crimes committed by adolescent offenders are often connected to alcohol and other drug use (Mulvey et al., 2010; National Institute of Justice, 1997; 2003). Among juvenile offenders, lifetime prevalence rates of alcohol and marijuana use have been found to be as high as 80 and $85 \%$ respectively, with $40 \%$ reporting alcohol use and $57 \%$ reporting marijuana use in the past 6 months (Mulvey et al., 2010). Furthermore, substance use is positively associated with the rate and severity of offending (Greenwood, 1992; Lipsey \& Derzon, 1998; Sealock et al., 1997). Perhaps most alarming is the relationship between substance use and violent crime that exists within this population (Mulvey et al., 2010). Alcohol use in particular has

(C) 2013 Elsevier Inc. All rights reserved.

*Corresponding author. Social Sciences Research Center, University of Rhode Island, 130 Flagg Rd., Kingston, RI 02881. Tel.: +1 401 874 4013; fax: +1 4018745562 . 
consistently been found to be associated with violent crime among incarcerated juveniles (Lennings et al., 2003).

\subsection{Ethnic differences in substance use}

Despite a clear connection between substance use and crimes, there is little understanding of the nature of substance use and its contributing factors across ethnic groups involved in the juvenile justice system (Feldstein Ewing et al., 2012). When compared to their White counterparts, minority adolescent offenders have been found to have lower rates of substance use and other risk factors but greater levels of impairment following their involvement with the justice system (Feldstein Ewing et al., 2011). It is important to look at factors that influence substance use, such as individual characteristics, across ethnic groups. For example, one recent study found that compared to White adolescents, higher lifetime substance use among Hispanic adolescents was mediated by negative expectancies and resistance self-efficacy (individual factors). Compared to White adolescents, lower lifetime substance use among Asian adolescents was mediated by positive and negative expectancies and resistance self-efficacy (individual factors) (Shih et al., 2010). Given these findings, treatment approaches that target substance use expectancies and self-efficacy with incarcerated adolescents, such as motivational interviewing (MI), should be investigated as they may differentially impact ethnic populations.

\subsection{Motivational interviewing with offenders}

One efficacious intervention for adolescent and adult offenders is MI (Feldstein \& Ginsburg, 2006; McMurran,2009). Studies with offenders have found that MI increases motivation and confidence toreduce substance use (Mendel \& Hipkins, 2002; Slavet et al., 2005;

Vanderberg,2003). Specifically among adolescent offenders, MI has been found to reduce negative treatment engagement, (Stein et al., 2006b), risky behaviors (Rosengard et al., 2007; Stein et al., 2006a; Stein et al., 2011), and substance use (Stein et al., 2011). Further, a group intervention of motivational enhancement therapy for detained adolescents found that self-efficacy is an important mechanism of change (Schmiege et al., 2009). Self efficacy is related to behavioral intentions, which significantly predicts later risky sexual behavior (Schmiege et al., 2009).

\subsection{Motivational interviewing with ethnic minority groups}

Approximately $61 \%$ of juvenile offenders in custody are members of an ethnic minority group; 28\% identify as Black, 19\% Hispanic/Latino, 2\% Asian, and 2\% Indian (Snyder \& Sickmund, 2006). Evidence supporting the efficacy of MI among ethnic minority groups remains mixed. Some studies have found support for the efficacy of MI with nonincarcerated Hispanic/Latino adults and adolescents whose primary substance was alcohol (Carroll et al., 2009; D'Amico et al., 2008). Furthermore, a meta-analysis conducted by Hettema et al. (2005) concluded that MI produces significantly larger effects for minority samples than for non-minority White samples. A more recent meta-analysis similarly found MI to be more successful with individuals from minority ethnic groups (Lundahl et al., 2010). Among minority ethnic adolescents, MI has produced reductions in binge drinking episodes, frequency of alcohol and marijuana use, affiliation with substance using peers, and alcohol related consequences (D'Amico et al., 2008; Gil et al., 2004; Schmiege et al., 2009; Walton, et al., 2010). The success MI among minority populations may be attributed to the person-centered approach of the intervention which may make it more compatible and attractive to groups who have experienced societal rejection (Lundahl et al., 2010).

However, results from other studies suggest that MI is not advantageous for African Americans (Befort et al., 2008; Lundahl et al., 2010; Tonigan et al., 2003) or Hispanic/ Latino Americans (Arroyo et al., 2003). Some suggest certain ethnic groups may want to 
receive help from an expert, may prefer client-therapist power differentials, or may not feel comfortable with a strong person-centered approach (Hays, 2009; Lopez Viets, 2007; Miller et al., 2010). Moreover, mechanisms of change may differ across ethnic groups. For example, one ethnic group may find community-oriented cognitions (e.g., ability to navigate peer influences) salient while another group may find individual-oriented internal cognitions (e.g., motivation for change) more important (Feldstein Ewing et al., 2011).

Such divergent findings suggest it is important to examine how treatment approaches such as MI may differentially impact substance use among ethnic populations in the juvenile justice system. The purpose of the current study was to investigate the influence of ethnicity on treatment in reducing alcohol and marijuana use among incarcerated adolescents after release. We know of no studies to date focusing on the potential moderating effects of ethnicity when examining the impact of MI on substance use for incarcerated youth. This represents an underserved and diverse population in great need. These data were collected as part of a parent study examining the impact of MI on substance use.

\section{Materials and methods}

\subsection{Participants}

The participants were recruited over a 5 year period at a state juvenile correctional facility in the Northeast (April, 2001 to March, 2006). Potential participants were identified immediately after adjudication if they were between the ages of 14 and 19 years (inclusive) and were sentenced to the facility for between 4 and 12 months (inclusive). Substance use inclusion criteria included: (1) in the year prior to incarceration they (1a) used marijuana or drank regularly (at least monthly), or ( $1 b$ ) they binge-drank ( $\geq 5$ standard drinks for boys; $\geq 4$ for girls) at least once; (2) they used marijuana or drank in the 4 weeks before the offense for which they were incarcerated; or (3) they used marijuana or drank in the 4 weeks before they were incarcerated.

There were 189 adolescents who completed the baseline assessment. Of these, 181 participants completed the 3-month post-release follow-up; five could not be located for follow-up, and three adolescents withdrew from the study prior to completion of the 3month follow-up. Most of the sample was male (85.7\%) with a mean age at baseline of 17.12 years (standard deviation, $S D=1.10$ ). In the previous year, most had a marijuana diagnosis (89\%; $16 \%$ abuse and $73 \%$ dependence), and over half (59\%) had an alcohol diagnosis (24\% abuse and 35\% dependence). The median number of times adolescents had been previously incarcerated was 2 .

There were no differences between those who completed the 3 month follow-up and those who did not on gender, ethnic status, or mother's education (a marker for socio-economic status). Adolescents who did not complete the 3-month follow-up were significantly older $(M=18.13, S D=0.90)$ than those who completed the follow-up $(M=17.07, S D=1.09)$, $t(187)=2.71, p<.01$. Adolescents in a controlled environment for $250 \%$ of days during the baseline or 3 month-follow-up period were removed from analyses $(n=19)$ and 15 participants did not identify with White, African-American, or Hispanic ethnic backgrounds, leaving a sample of $N=147$ [note: the term Hispanic was used consistent with the United States Census, which uses Hispanic to refer to "a person of Cuban, Mexican, Puerto Rican, South or Central American, or other Spanish culture or origin regardless of race" (Rastogi et al., 2011)]. Of this sample, 48 were White, 51 were Hispanic, and 48 were African American. 


\subsection{Procedures}

Initial screening was based on record review for age and sentence length, followed by screening the potential participant for the substance use criteria described above. Institutional review board approval for all procedures was obtained as well as consent from legal guardians and assent from adolescents (adolescents 18 years or older provided consent). Guardians and adolescents were given the limits of confidentiality. (i.e., except for plans to escape, hurt self or others, reports of child abuse and/or neglect). After the participant was recruited, the baseline assessment was conducted, and the adolescent was randomly assigned to a single session of individual treatment, either MI or relaxation therapy (RT). After treatment, the adolescents enrolled in standard facility substance use programming (see Stein et al., 2011 for a full description of the facility and the standard substance use programming). Approximately 2 weeks before facility discharge, they received booster intervention (MI or RT). Research staff blind to treatment assignment conducted a follow-up assessment 3 months after release from the facility. Randomization was accomplished via random numbers table in advance and placed in an envelope by the project coordinator. Following baseline assessment, treatment providers opened the envelope to learn of intervention assignment.

\subsection{Assessment}

Trained bachelor's (BA/BS) or master's (MA)-level research assistantsconducted the 60-90 minutes of private interview assessments. Interview format was used dueto concerns about reading levels of the participants. Research assistants' training andsupervision are detailed elsewhere (Stein et al., 2006a; Stein et al., 2011). Record reviews were completed following completion of the baseline assessments. Follow-up assessments were conducted 3 months after release from the facility with adolescents receiving a $\$ 60$ gift certificate and a $\$ 10$ bonus if they completed the interview within 1 week of the scheduled date.

\subsection{Study interventions}

Shortly after the baseline assessment, adolescents were randomly assigned to and received intervention (MI or RT), in order to prepare them for the standard facility substance use programming. Research counselors were two men and two women, all four were Caucasian, one had an MA degree and three had BA/BS degrees. Each research counselor conducted both intervention types and received about 56 hours of manualized training with 2 hours of group and 1 hour of individual supervision per week. To maintain fidelity to treatment, in vivo observations were conducted by a licensed clinical psychologist. Interventions were about 90 minutes at baseline and about 60 minutes at booster.

2.4.1. Motivational interviewing-Miller and Rollnick's (2002) principles of MI were the basis of the intervention protocol. The protocol included developing rapport, exploration of motivation (pros and cons), personalized assessment feedback, imagining the future with and without change, and establishing goals. Handouts were provided, including goal sheets. The intervention focused on reducing alcohol and/or marijuana use and associated risky behaviors and consequences of use (e.g., injuries while drunk/high).

2.4.2. Relaxation training-RT was designed to control for the effects of attending an individual intervention. Participants were instructed in progressive muscle relaxation and use of imagery to produce a sense of calm. Adolescents received feedback in the use of the techniques, and they were provided handouts on progressive muscle relaxation. Research counselors maintained rapport and provided general advice to stop risky activities involving alcohol and marijuana use. The rationale for RT was that these techniques can reduce the 
stress that can lead to substance use such as alcohol and marijuana; thus, these techniques may lead to reduced substance use and their associated risky behaviors and consequences.

\subsection{Measures}

2.5.1. Record review-Adolescents were informed at the start of the study that records would be reviewed at baseline assessment to verify self-reports of alcohol/marijuana use and illegal activity.

2.5.2. Background questionnaire-At baseline assessment, socio-demographic information was recorded including age, gender, ethnicity, number of years of school completed, and parent/guardian educational level.

2.5.3. Timeline followback (TLFB)-Time-line follow-back is a calendar-assisted approach that measures participants' recollection of their substance use over a specified period of time (Sobell \& Sobell, 1992). It has been used to assess substance use (Bardone et al., 2000; Midanik et al., 1998; Sobell \& Sobell, 1992; Stein et al., 2011). TLFB has been shown to have excellent reliability ( $\alpha$;'s $=.79$ to .98 ; Sobell et al., 1979) and strong content, criterion, and construct validity. A 90 day TLFB measuring alcohol and marijuana use was collected at baseline and at follow-up after release.

\subsection{Analyses}

Analysis of covariance (ANCOVA) was used to determine the impact of ethnicity on treatment to reduce alcohol and marijuana use. Tests were conducted using Bonferroni hypothesis-wide adjusted alpha levels of .025 (.05/2 dependent variables per substance). Alcohol and marijuana use variables were log-transformed to correct skewness (no transformation was needed for percentage of days used marijuana). Dependent marijuana use variables (DV's) at 3-months post-release assessment were average number of joints smoked on smoking days (MJSD) and percentage of days used marijuana (PDM). Since the focus of this study is on heavy drinking, total number of drinks on heavy drinking days (NDHD) and percentage of heavy drinking days (PHDD) were alcohol use dependent variables. Heavy drinking was defined as four or more for girls and five or more for boys. For each ANCOVA, the covariate was the corresponding baseline measure of the DV, and the independent variables (IVs) were intervention condition and ethnicity along with the interaction of intervention condition and ethnicity. The baseline value of the DV was included in analyses to balance groups on covariates. Adjusting for these covariates improves the efficiency of the analysis and provides stronger evidence of the treatment effect (Assmann et al., 2000).

\section{Results}

No significant differences were found between treatment groups on relevant baseline variables including gender, age, ethnicity, or mother's education level. Randomization of ethnic groups to treatment was equal $X(2)=2.317, p=.314$. Significant differences were found between ethnic groups at baseline for the transformed alcohol variables. White teens drank significantly more total drinks on heavy drinking days $(M=2.13,95 \%$, CI [1.94, 2.33]) than African American teens $(M=1.58,95 \%, \mathrm{CI}[1.32,1.85])$ and had a higher percentage of heavy drinking days ( $M=1.03,95 \%, \mathrm{CI}[.86,1.20])$ than African American teens $(M=.4586,95 \%, \mathrm{CI}[.30, .62])$ and Hispanic teens $(M=.72,95 \%$, CI $[.55, .89])$. No other differences were significant between ethnic groups. No significant results were found for any of the marijuana TLFB variables in terms of main outcomes or interaction effects so these analyses are not presented. Means and standard deviations for baseline alcohol and marijuana use variables are presented in Table 1. 
Significant main effects and the interactions are presented in Table 2. Significant main effects were found for ethnicity on total number of drinks on heavy drinking days (NDHD), but no main effects were found for ethnicity on percentage of heavy drinking days (PHDD). There was a significant treatment by ethnicity interaction for total number of drinks on heavy drinking days (NDHD) and percentage of heavy drinking days (PHDD) (Figs. 1 and 2).

Table 3 presents results of simple-effects tests for significant interactions. Hispanic adolescents who received MI significantly decreased total number of drinks on heavy drinking days (NDHD) and percentage of heavy drinking days (PHDD) as compared to Hispanic adolescents who received RT.

\section{Discussion}

The present study investigated the moderating effects of ethnicity on treatment outcomes for substance-using, incarcerated adolescents. Significant treatment by ethnicity interactions were found. Hispanic adolescents who received MI reported a significant decrease in the total number of drinks consumed on heavy drinking days and the percentage of heavy drinking days compared to Hispanic adolescents who received RT. These findings are consistent with earlier research that found MI to be efficacious among Hispanic populations (Carroll et al., 2009; D'Amico et al., 2008; Drenner, 2009; Henslee et al., 2009; Ritamarie, 2010; Robles et al., 2004).

Contrary to expectations, ethnicity was not found to moderate the effects of treatment on marijuana use outcomes. This lack of significant findings may result from both treatments working equally well for marijuana. However, the present study findings are consistent with previous research that found MI to be differentially effective for Hispanic individuals with alcohol use problems (Carroll et al., 2009; D'Amico et al., 2008). Additional research is needed to further elucidate the differential impact of MI on alcohol and marijuana use outcomes among Hispanic populations.

The success of MI with Hispanic populations has been hypothesized to result from the congruence between MI principles and cultural-specific Hispanic values. Añez et al. (2008) propose that personalismo (preference for a relationship with individuals rather than institutions), respeto (respect), and confianza (trust and intimacy in a relationship) are significant constructs in the daily lives of Hispanic culture. The compatibility between these constructs and MI principles suggest that MI is a culturally congruent treatment for this ethnic minority population. Further, some findings indicate that Hispanic adolescent substance use is mediated by expectancies and self-efficacy (Shih et al., 2010). Since MI often targets negative and positive expectancies for substance use and aims to improve selfefficacy, this intervention may be efficacious for juvenile justice-involved Hispanic adolescents.

Findings should be interpreted with caution due to several limitations. The sample consisted of incarcerated adolescents, most of whom were male. This may limit the generalizability of the findings. Also, the study did not include measures of acculturation, which may have helped to refine the ethnic categories. Further, the study relied primarily on self-report methods; however, research suggests such methods are generally accurate (Babor et al., 2002). In addition, Stein et al. (2011) demonstrated that this sample generally reported accurately when examining self-report against biological testing and record review. Although the present study is limited by the relatively brief follow-up period, this is common when treatment effects are initially being established. Furthermore, baseline differences between groups, even when controlled statistically, can affect outcomes. For example, African American youths drank less at baseline and thus have much less room to 
improve with treatment, making differential treatment effects difficult to detect. Future research may consider replicating these findings with a larger sample that includes more females and a longer follow-up period. Also, these are secondary analyses that need replication in a study powered to study interactive effects. Despite these limitations, the present study findings remain significant as they are among the first to suggest that $\mathrm{MI}$ is an efficacious treatment for an ethnic minority juvenile justice-involved population in need of evidence-based interventions.

\section{Acknowledgments}

This work was supported by grants from the National Institute on Drug Abuse, R01-13375 (PI, Stein) and the National Institute on Drug Abuse/National Institute on Alcohol Abuse and Alcoholism R01-18851 (PI, Stein).

\section{References}

Añez LM, Silva MA, Paris M, Bedregal LE. Engaging Latinos through the integration of cultural values and motivational interviewing principles. Professional Psychology: Research and Practice. 2008; 39:153-159.

Arroyo JA, Miller WR, Tonigan JS. The influence of Hispanic ethnicity on long-term outcome in three alcohol treatment modalities. Journal of Studies on Alcohol and Drugs. 2003; 64:98-104.

Assmann SF, Pocock SJ, Enos LE, Kasten LE. Subgroup analysis and other (mis)uses of baseline data in clinical trials. The Lancet. 2000; 355:1064-1069.

Babor TF, Webb C, Burleson JA, Kaminer Y. Subtypes of classifying adolescents with marijuana use disorders: Construct validity and clinical implications. Addiction. 2002; 97:58-69. [PubMed: 12460129]

Bardone AM, Krahn DD, Goodman BM, Searless JS. Using interactive voice response technology and timeline follow-back methodology in studying binge eating and drinking behavior: Different answers to different forms of the same question? Addictive Behaviors. 2000; 25:1-11. [PubMed: 10708315]

Befort CA, Nollen N, Ellerbeck EF, Sullivan DK, Thomas JL, Ahluwalia JS. Motivational interviewing fails to improve outcomes of a behavioral weight loss program for obese African American women: A pilot randomized trial. Journal of Behavioral Medicine. 2008; 31:367-377. [PubMed: 18587639]

Carroll KM, Martino S, Ball SA, Nich C, Frankforter T, Anez LM, Farentinos C. A multisite randomized effectiveness trial of motivational enhancement therapy for Spanish-speaking substance users. Journal of Consulting and Clinical Psychology. 2009; 77:993-999. [PubMed: 19803579]

Cohen, J. Statistical Power Analysis for the Behavioral Sciences. 2nd. Hillsdale: Lawrence Erlbaum Associates; 1988.

D'Amico EJ, Miles JNV, Stern SA, Meredith LS. Brief motivational interviewing for teens at risk of substance use consequences: A randomized pilot study in a primary care clinic. Journal of Substance Abuse Treatment. 2008; 35:53-61. [PubMed: 18037603]

Drenner KL. If we call them, will they change? Feasibility of a telephone adaption of motivational interviewing aimed at parents to reduce television time among 2nd and 4th grade children. Dissertation Abstracts International. 2009; 70(3-B):1931B.

Feldstein Ewing SW, Venner KL, Mead HK, Bryan AD. Exploring racial/ethnic differences in substance use: A preliminary theory-based investigation with juvenile justice-involved youth. BMC Pediatrics. 2011; 11:71-81. [PubMed: 21846356]

Feldstein Ewing SW, Wray AM, Mead HK, Adams SK. Two approaches to tailoring treatment for cultural minority adolescents. Journal of Substance Abuse Treatment. 2012; 43:190-203. [PubMed: 22301086]

Feldstein SW, Ginsburg JID. Motivational interviewing with dually diagnosed adolescents in juvenile justice settings. Brief Treatment and Crisis Intervention. 2006; 6:218-233. 
Gil AG, Wagner EF, Tubman JG. Culturally sensitive substance abuse intervention for Hispanic and African American adolescents: Empirical examples from the Alcohol Treatment Targeting Adolescents in Need (ATTAIN) Project. Addiction. 2004; 99:140-150. [PubMed: 15488112]

Greenwood PW. Substance abuse problems among high-risk youth and potential intervention. Crime and Delinquency. 1992; 38:444-458.

Hays PA. Integrating evidence-based practice, cognitive-behavior therapy, and multicultural therapy: Ten steps for culturally competent practice. Professional Psychology: Research and Practice. 2009; 40:354-360.

Henslee AM, Schumacher JA, Holloman G, Coffey SF. Unresolved PTSD in a Hispanic woman presenting with test anxiety. Clinical Case Studies. 2009; 8:340-350.

Hettema J, Steele J, Miller WR. Motivatonal interviewing. Annual Review of Clinical Psychology. 2005; 1:91-111.

Lennings CJ, Copeland J, Howard J. Substance use patterns of young offenders and violent crime. Aggressive Behavior. 2003; 29:414-422.

Lipsey, MW.; Derzon, JH. Predictors of violent or serious delinquency in adolescence and early adulthood: A synthesis of longitudinal research. In: Loeber, R.; Farrington, DP., editors. Serious and Violent Offenders. Thousand Oaks, CA: Sage Publications, Inc; 1998. p. 313-345.

Lopez Viets V. CRAFT: Helping Latino families concerned about a loved one. Alcoholism Treatment Quarterly. 2007; 25:111-123.

Lundahl BW, Kunz C, Brownell C, Tollefson D, Burke BL. A meta-analysis of motivational interviewing: Twenty-five years of empirical studies. Research on Social Work Practice. 2010; 20:137-160.

McMurran M. Motivational interviewing with offenders: A systematic review. Legal and Criminological Psychology. 2009; 14:83-100.

Mendel E, Hipkins J. Motivating learning disabled offenders with alcohol-related problems: A pilot study. British Journal of Learning Disabilities. 2002; 30:153-158.

Midanik LT, Hines AM, Barrett DC, Paul JP, Crosby GM, Stall RD. Self-reports of alcohol use, drug use and sexual behavior: Expanding the timeline follow-back technique. Journal of Studies on Alcohol. 1998; 59:681-689. [PubMed: 9811089]

Miller ST, Marolen KN, Beech BM. Perceptions of physical activity and motivation interviewing among rural African-American women with type 2 diabetes. Women's Health Issues. 2010; 20:4349. [PubMed: 19944621]

Miller, WR.; Rollnick, S. Motivational interviewing: Preparing people for change. 2nd. New York: The Guildord Press; 2002.

Mulvey, EP.; Schubert, CA.; Chassin, L. Substance use and delinquent behavior among serious adolescent offenders Juvenile Justice Bulletin. Washington, D.C: U.S. Department of Justice; 2010.

National Institute of Justice. 1997 annual report on adult and juvenile arrestees. Washington, DC: U.S. Department of Justice; 1997.

National Institute of Justice. 2000 arrestee drug abuse monitoring: Annual report (NCJ 193013). Washington, DC: U.S. Department of Justice; 2003.

Rastogi, S.; Johnson, TD.; Hoeffel, EM.; Drewery, MP. The Hispanic Population: 2010, Census Briefs, U.S. Census Bureau. 2011. Retrieved February 20, 2012, from. http://www.census.gov/ prod/cen2010/briefs/c2010br-06.pdf

Ritamarie J. Effects of parent-focused media interventions on body mass index, waist size, selfperception, family eating habits, and family activity habits in overweight Hispanic children. Dissertation Abstracts International. 2010; 70:4087B.

Robles RR, Reyes JC, Colón HM, Sahai H, Marrero CA, Matos TD, Shepard EW. Effects of combined counseling and case management to reduce HIV risk behaviors among Hispanic drug injectors in Puerto Rico: A randomized controlled study. Journal of Substance Abuse Treatment. 2004; 27:145-152. [PubMed: 15450647]

Rosengard C, Stein LAR, Barnett NP, et al. Randomized clinical trial of motivational enhancement of substance use treatment among incarcerated adolescents: Post-release condom non-use. Journal of HIV/AIDS Prevention in Children \& Youth. 2007; 8:45-64. [PubMed: 19809580] 
Schmiege SJ, Broaddus MR, Levin M, Bryan AD. Randomized trial of group interventions to reduce HIV/STD risk and change theoretical mediators among detained adolescents. Journal of Consulting and Clinical Psychology. 2009; 77:38-50. [PubMed: 19170452]

Sealock MD, Gottfredson DC, Gallagher CA. Drug treatment for juvenile offenders: Some good and bad news. Journal of Research in Crime and Delinquency. 1997; 34:210-236.

Shih RA, Miles JNV, Tucker JS, Zhou AJ, D'Amico EJ. Racial/ethnic differences in adolescent substance use: Mediation by individual, family, and school factors. Journal of Studies on Alcohol and Drugs. 2010; 71:640-651. [PubMed: 20731969]

Slavet JD, Stein LAR, Klein J. Piloting the family check up with incarcerated adolescents and their parents. Psychological Services. 2005; 2:123-132. [PubMed: 19756250]

Snyder, HN.; Sickmund, M. Juvenile offenders and victims: 2006 National Report. Washington, DC: U.S Department of Justice, Office of Justice Programs, Office of Juvenile Justice and Delinquency Prevention; 2006.

Sobell LC, Maisto SA, Sobell MB, Cooper AM. Reliability of alcohol abusers' self-reports of drinking behavior. Behavior Research and Therapy. 1979; 17:157-160.

Sobell, LC.; Sobell, MB. Timeline follow-back: A technique for assessing self-reported alcohol consumption. In: Allen, J.; Litten, RZ., editors. Measuring alcohol consumption: Psychosocial and biological methods. Totowa, NJ: Humana Press Inc.; 1992. p. 41-72.

Stein LAR, Colby SM, Barnett NP, Monti PM, Golembeske C, Lebeau-Craven R. Effects of motivational interviewing for incarcerated adolescents on driving under the influence after release. The American Journal on Addictions. 2006; 15(Suppl 1):50-57. [PubMed: 17182420]

Stein LAR, Lebeau R, Colby S, Barnett NP, Golembeske C, Monti P. Motivational interviewing for incarcerated adolescents: Effects of depressive symptoms on reducing alcohol and marijuana use after release. Journal of Studies on Alcohol and Drugs. 2011; 72:497-506. [PubMed: 21513687]

Stein LAR, Monti PM, Colby SM, Barnett NP, Golembeske C, Lebeau-Craven R, Miranda R. Enhancing substance abuse treatment engagement in incarcerated adolescents. Psychological Services. 2006; 3:25-34. [PubMed: 20617117]

Tonigan JS, Miller WR, Daughtery M, Carrol RL. Project MATCH treatment participation and outcome by race: A restrospective analysis of African American treatment response. Alcoholism, Clinical and Experimental Research. 2003; 27(Suppl):A159.

Vanderberg SA. Motivational Interviewing as a pre-cursor to a substance abuse program for offenders. Dissertation Abstracts International. 2003; 63:4354B.

Walton MA, Chermack ST, Shope JT, Bingham CR, Zimmerman MA, Blow FC, et al. Effects of a brief intervention for reducing violence and alcohol misuse among adolescents: A randomized controlled trial. JAMA : The Journal of the American Medical Association. 2010; 304:527-535. [PubMed: 20682932] 


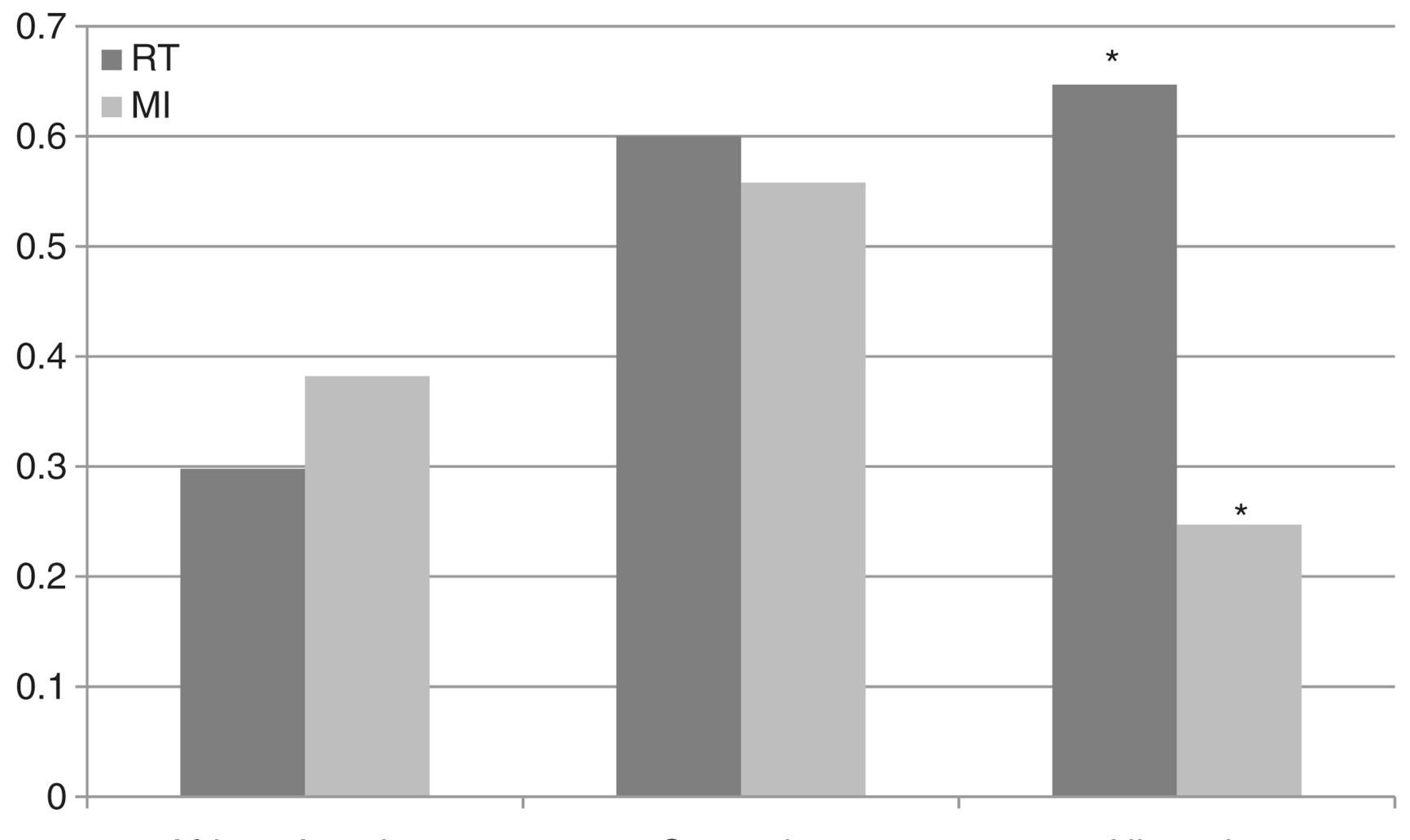

African American

Caucasian

Hispanic

Fig. 1.

Treatment by ethnicity interaction for percentage of heavy drinking days (PHDD; logtransformed) at 3-month follow-up. RT = relaxation therapy; MI = motivational interviewing; $* p<.05$. 


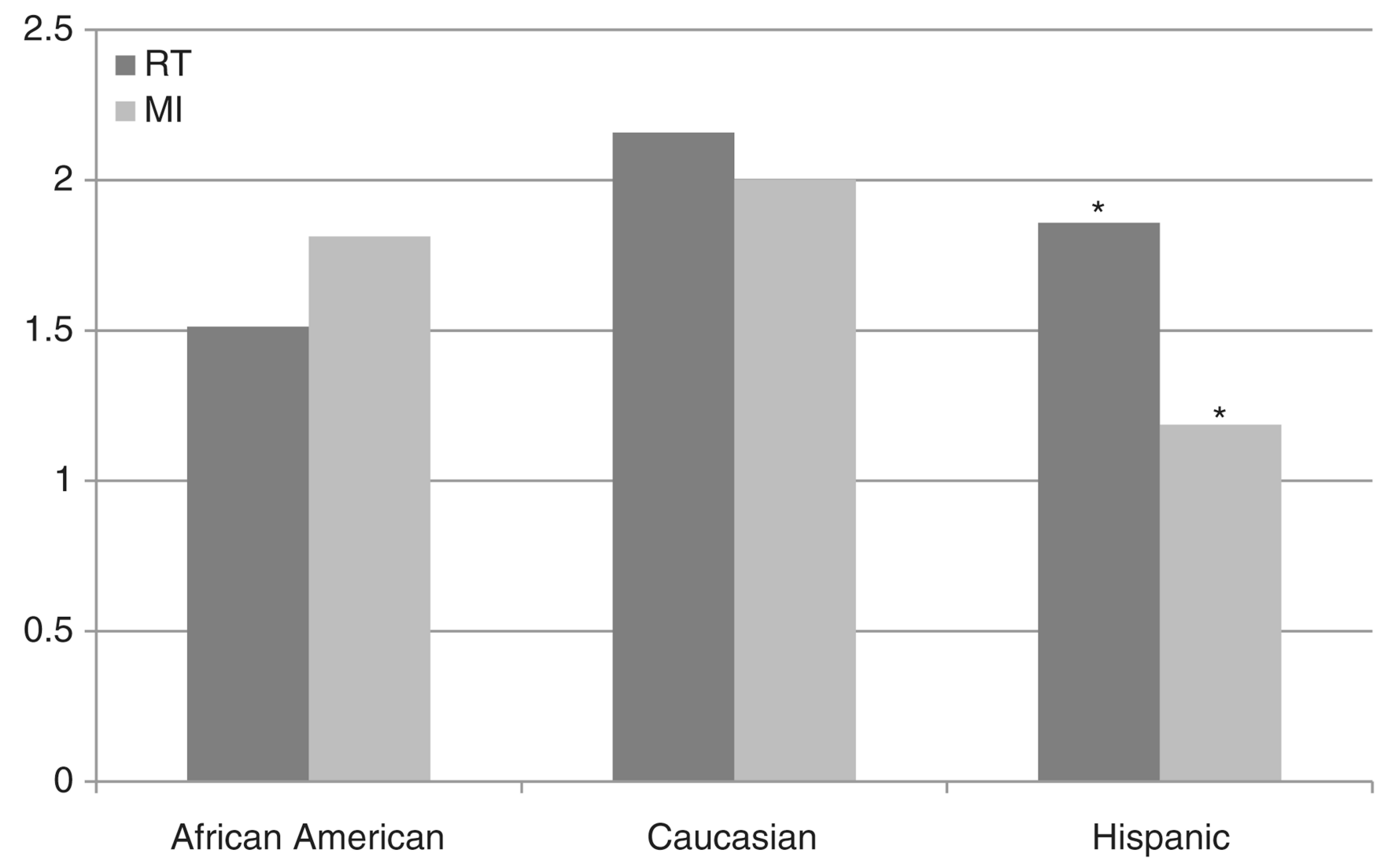

Fig. 2.

Treatment by ethnicity interaction for total number of drinks on heavy drinking days (NDHD; log-transformed) at 3-month follow-up. RT = relaxation therapy; $\mathrm{MI}=$ motivational interviewing; $* p<.05$. 


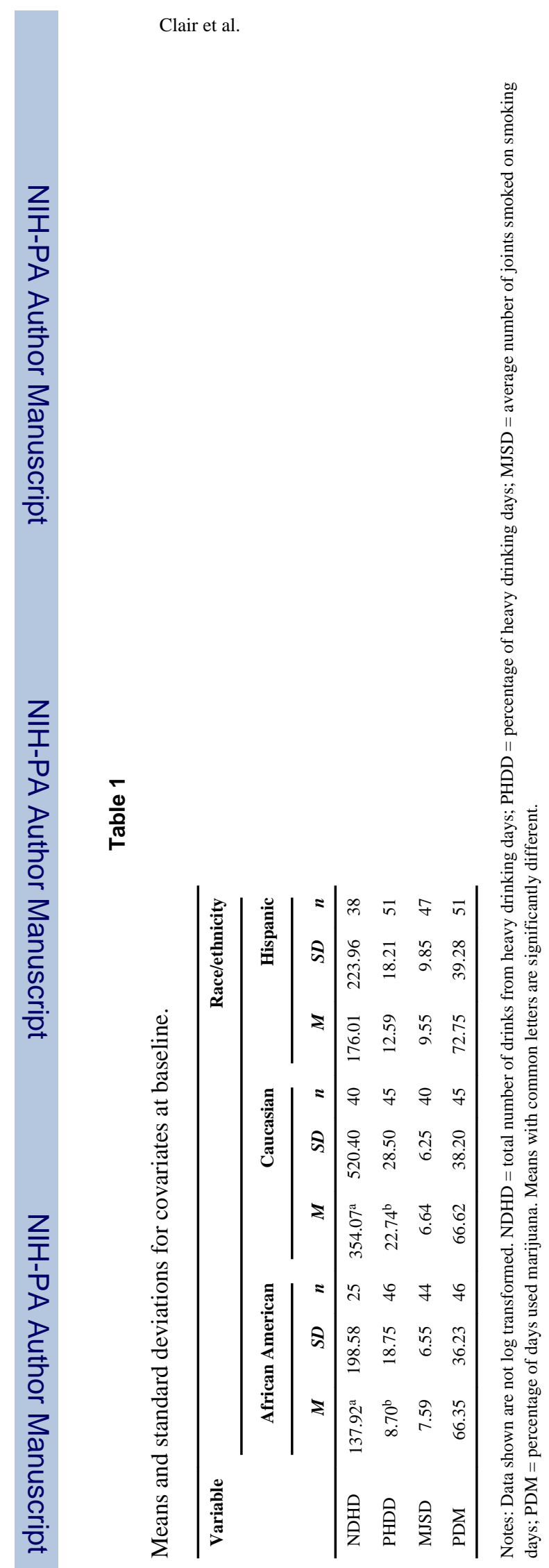

J Subst Abuse Treat. Author manuscript; available in PMC 2014 October 01. 
Table 3

Follow-up tests comparing ethnicity within treatments.

\begin{tabular}{clll}
\hline Variable & MI vs. RT & & \\
\cline { 2 - 4 } & African American & Caucasian & Hispanic \\
\hline NDHD & & & \\
$F^{a}$ & $.943(1,60), p \geq .335$ & $.640(1,60), p \geq .427$ & $10.27(1,60) p \leq .05$ \\
$\eta^{2}$ & $.015^{b}$ & $.011^{b}$ & $.146^{c}$ \\
PHDD & & & \\
$F^{a}$ & $.364(1,140), p \geq .547$ & $.097(1,140), p \geq .756$ & $9.314(1,140), \mathrm{p} \leq .003$ \\
$\eta^{2}$ & $.003^{b}$ & $.001^{b}$ & $.062^{d}$ \\
\hline
\end{tabular}

Notes: $\eta^{2}$ is partial $\eta^{2}$; MI = motivational interviewing; RT = relaxation therapy; NDHD = total number of drinks from heavy drinking days; $\mathrm{PHDD}=$ percentage of heavy drinking days.

${ }^{a} F$ statistic provided with degrees of freedom and associated p levels.

${ }^{b}$ Small effect size (Cohen, 1988).

$c$ Large effect size.

$d$ Medium effect size. 